\title{
Placement of percutaneous pedicle screws without imaging guidance
}

\author{
Ciaran J. Powers, M.D., Ph.D., Vinod K. Podichetty, M.D., M.S., \\ AND Robert E. ISAACS, M.D. \\ Division of Neurosurgery, Department of Surgery, Duke University Medical Center, Durham, North \\ Carolina; and Spine Institute, Cleveland Clinic, Weston, Florida
}

\begin{abstract}
$\checkmark$ Pedicle screw (PS) instrumentation provides an exceptionally rigid construct to promote fusion in cases of spinal trauma and degenerative disease. Although the safety of traditional open techniques for PS placement has been well documented, there are no large series in the literature in which the safety of percutaneously placed PSs has been examined. Because the advantages of minimally invasive spine surgery are becoming more widely recognized, especially in regard to the lessening of morbidity caused by pain and blood loss, there will be a greater demand for spine surgeons to place PSs percutaneously. During a 2-year period, the authors placed 287 PSs percutaneously with the aid of intraoperative fluoroscopy. Only one of these screws was later found to have breached the spinal canal, yielding a breach rate of $0.35 \%$ for percutaneously placed PSs (one of 287).
\end{abstract}

\section{KEY WORDS • minimally invasive surgery - minimally invasive spinal fusion • lumbar fusion - lumbosacral fusion - percutaneous surgery • pedicle screw}

$\mathrm{E}$ ARLY use was made of percutaneous PS fixation in cases of instability from acute trauma or osteomyelitis. Magerl ${ }^{13}$ began using PSs to secure an external spinal fixation system in 1977 . This system of external fixation in which percutaneous PSs are used had been developed to improve on the conventional treatment of spinal instability with Harrington rods, which required fusion of at least five vertebrae. Although Magerl did not recommend the use of percutaneous PSs for degenerative disorders, others soon began to use the broader applications of this treatment. The widespread use of percutaneous PS fixation has led to the development of more sophisticated minimally invasive instrumentation techniques, such as that described by Foley and Gupta ${ }^{2}$ in 2002. One of the major advantages of currently available systems over previous percutaneous techniques is that the connecting rods are placed under the muscle fascia in a fashion designed to minimize collateral tissue damage.

Early results had indicated that although percutaneous PS instrumentation remains a viable option, the breach rate remains higher than for PSs placed in an open fashion. In a study of human cadavers conducted in 1999, Wiesner, et al. ${ }^{18}$ reported a $10 \%$ misplacement rate of 360 PSs, the majority of which were medial. One year later, Wiesner and colleagues ${ }^{19}$ reported their clinical results in regard to misplaced percutaneous PSs, again noting a reasonably high

Abbreviations used in this paper: $\mathrm{AP}=$ anteroposterior; $\mathrm{CT}=$ computerized tomography; PS = pedicle screw. breach rate (6.6\%), as confirmed on CT scanning. This is thought to be especially true at $\mathrm{S}-1$, where the authors found a $12 \%$ breach rate.

Although several groups have presented data at the American Association of Neurological Surgeons/Congress of Neurological Surgeons Section on Disorders of the Spine and Peripheral Nerves Annual Meetings in 2003 (RP Nockels, unpublished data) and 2004 (MJ Perez-Cruet, et al., unpublished data) revealing an effective breach rate of $4 \%$ or lower in small numbers of percutaneous PSs, and we have published the results of our early experience with percutaneous screw placement, ${ }^{5}$ no recent study has specifically addressed the PS malpositioning rate in a large clinical series. We present here a series of patients in whom 287 percutaneous PSs were placed, with our goal being to determine the safety of placement in a clinical setting, specifically with regard to S-1 (Fig. 1).

\section{Clinical Material and Methods}

Between October 2002 and December 2005, 67 patients underwent percutaneous placement of PS instrumentation without imaging guidance. These procedures were performed at two hospitals (Cleveland Clinic in Weston, Florida, and Duke University Medical Center in Durham, North Carolina) under the guidance of the senior surgeon (R.E.I.). Indications varied from degenerative disease of the spine to trauma, and included the following: adjacent-level disease, burst fracture, chance fracture, discogenic low-back pain, failed-back syndrome, fracture-dislocation, multiple recur- 


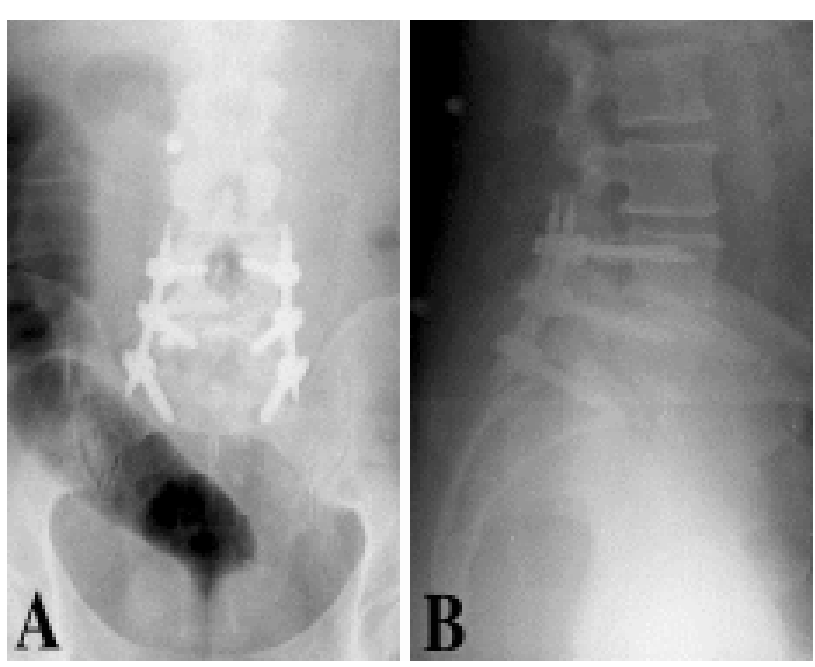

FIG. 1. Postoperative AP (A) and lateral (B) x-ray films showing the correct location and angulation of a three-level percutaneous PS placement in the distal lumbosacral spine.

rent disc herniations (at the same level), nonunion, postlaminectomy instability, recurrent thoracic disc, sagittal imbalance, scoliosis, and spondylolisthesis.

All screws were placed with the aid of only one fluoroscope in a standard operating room environment. During this period, all but one patient who underwent placement of standard open PS instrumentation required either removal of previously implanted spinal hardware (for adjacent-level disease) or placement of more than three consecutive PSs in adjacent levels of the spine. The exception was a patient with spontaneous, multilevel, Candida discitis that required an open, radical debridement. Until recently, percutaneous PS instrumentation was limited to three-level constructs, and therefore this study in effect addresses the safety of the placement of PS instrumentation in four- to six-pedicle-screw constructs. The number and distribution of PSs placed according to spinal level are represented in Fig. 2.

The choice of routine postoperative imaging modality was based somewhat on the patient's disease process, but in general this included static x-ray films for comparison with dynamic films obtained at 6 weeks, and then subsequent dynamic films obtained at later visits $(3,6,12$, and 24 months). For many patients, a routine CT scan was ordered at their initial clinic visit, or postoperatively to assess adequacy of fusion. In any patient with a new radicular complaint or new neurological deficit, a CT scan was performed at the time of presentation.

\section{Standard Percutaneous Screw Placement Technique}

Patients were placed prone on a Jackson table and positioned to improve sagittal balance. Fluoroscopy was used to determine the Ferguson angle, thereby delineating the superior endplate of the level that would receive instrumentation. The radiographic projection of these locations was marked on the skin. A true AP view was confirmed by centering the spinous process between the pedicles. These angles were recorded on the fluoroscope, and the maneuver was repeated later in the procedure. In addition, we obtained 10 to $25^{\circ}$ off-angle oblique images through the pedi- cles ("owl's eye" views), which were also marked on the skin and recorded on the fluoroscope. The off-angle Ferguson views were then used to place the K-wires.

With the fluoroscope angled in line with the target pedicle (10-25 off the angle of the straight AP image, depending on the level of approach), a Jamsheedi needle was inserted at the radiographic center of the pedicle. Once the location of the tip of this device was confirmed, the Jamsheedi needle was aligned to the beam axis of the fluoroscope. The K-wire was then advanced through the device and delivered approximately $1.5 \mathrm{~cm}$ into the pedicle. The angle of the fluoroscope was changed to a subsequent pedicle orientation and the process was repeated until all $\mathrm{K}$-wires were inserted with guidance from these off-angle oblique Ferguson views. After placement of each K-wire, the Jamsheedi needle was removed and a repeated x-ray film was acquired to confirm that the K-wire remained within the radiographic center portion of the pedicle. If it did not, the K-wire was removed and the process was repeated.

After all the K-wires had been inserted and their position confirmed using both off-angle oblique images and a straight AP view, we converted to a lateral view. The $\mathrm{K}$-wires were then advanced to the middle of the vertebral body after confirming their trajectory through the pedicle on the lateral image and adjusting their path as needed. With the K-wires in position, placement of PS instrumentation followed. After dilating the paraspinal musculature, the cortical bone of the pedicle was breached, typically with a cannulated awl. A path through the pedicle was made with a cannulated tap, and a cannulated screw followed. Once the screw was noted to be within the vertebral body on lateral imaging, the K-wire was removed. After this was done, the screw was advanced until its head was adjacent to but not impinging on the posterior elements of the spine.

\section{Results}

Between October 2002 and December 2005, 67 patients underwent an operation performed by the senior surgeon (R.E.I.) that was facilitated by the placement of percutaneous PS instrumentation without imaging guidance. This included a varying distribution of four-, five-, and six-level constructs (in 54, seven, and six patients, respectively) based on the disease process, and initially on the technical limitations of the instrumentation systems. No surgery was converted to an open procedure. In no case were we unable to place instrumentation in a pedicle due to our inability to visualize the structure, and in only one case (see later discussion) was revision of a screw required.

In total, 287 percutaneous screws were placed as outlined earlier, and these serve as the basis for this study. The screws were distributed between T-7 and S-1, with nearly one in five being placed in the thoracic or thoracolumbar spine. We used the Sextant system in approximately $70.1 \%$ of the screws placed, although nine different percutaneous systems were used in the course of this study. These systems included the following: Dynaloc, M-8, and Sextant (Medtronic Sofamor Danek, Memphis, TN); Pathfinder (Spinal Concepts, Inc. [a division of Abbott Spine, Austin, TX]); Spherix DBR and SBR (NuVasive, Inc., San Diego, $\mathrm{CA})$; Title (Endius, Inc., Plainville, MA); and Viper and VSP (DePuy Spine [a division of Johnson \& Johnson, Arlington, TX]). 


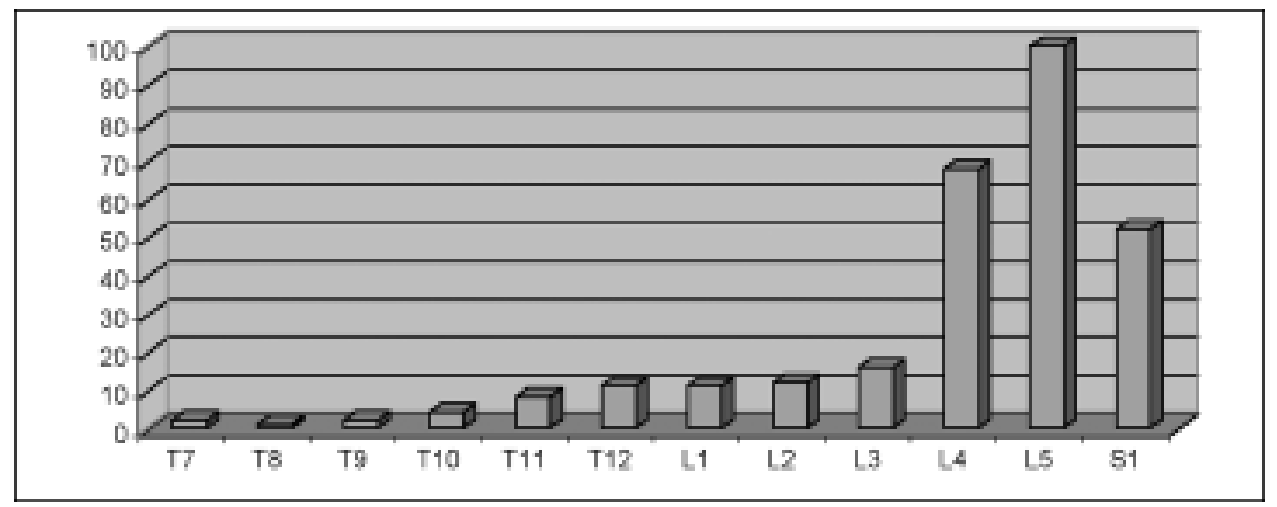

FIG. 2. Bar graph showing distribution of PSs based on spinal level. Labels on the $\mathrm{x}$ axis represent the vertebral level, and those on the y axis indicate the number of screws placed.

Screws were placed for various reasons, comprising essentially the entire spectrum of indications for posterior segmental instrumentation. This range included primarily degenerative disease but also reconstruction in patients undergoing surgery for trauma, tumor, and various other forms of spinal instability. The degenerative disease category can be further subdivided into multiple categories of patients with overt spinal deformity, including isthmic spondylolisthesis, degenerative spondylolisthesis, and postlaminectomy instability as well as patients undergoing surgery for flat back deformity and scoliosis. Follow-up CT scans were obtained in a number of patients as part of various protocols and can therefore be used to determine screw position in the majority of cases. Furthermore, CT scans were obtained for evaluation of a construct complication in any patient who displayed new radiculopathy or a neurological deficit, or whose condition failed to improve or worsened at any time during postoperative recovery. In 35 patients CT scans were available for review by an independent radiologist; these cases represented 148 (51.6\%) of the 287 screws placed in this series.

No patient experienced a worsening radiculopathy or new neurological deficit that could be attributed to screw malposition. One screw was found to have breached medially on routine CT scans obtained in a patient who experienced a continuation, but no worsening, of L-5 motor weakness after having undergone two-level interbody fusion. Interestingly, this patient took no narcotic medication after leaving the hospital and, based on her plain x-ray films, no significant medialization of the screw track was appreciated (Fig. 3). Although the breach was found within 2 months of surgery, the patient underwent elective L-4 screw repositioning approximately 6 months after the initial surgical intervention. The repositioning surgery was subsequently complicated by a superficial wound infection. Two years after the initial surgery, her hardware remains in place, and she no longer receives antibiotic drugs. In one other case, the concern for a slightly malpositioned screw on initial CT scanning was eliminated on repeated scanning performed with different gantry settings. Our breach rate of $0.35 \%$ (that is, one in 287 screws) includes no medial breaches in $50 \mathrm{~S}-1$ screw placements, and no significant misplacement in the thoracic or thoracolumbar spine.

Except in the patient noted in the preceding paragraph, in no other case has a hardware failure or screw malposition been noted. Specifically, no screw dislodgement, broken screws, or evidence for screw lucency has been seen in the remainder of these patients. In no other case has a wound or hardware infection (either superficial or deep) been appreciated or treated.

\section{Discussion}

Low-back pain is one of the most common conditions causing patients to seek medical care in the US. ${ }^{14}$ Although the majority of these patients improve with conservative management, some will require fusion of one or more spinal segments to treat their condition adequately. Lumbar fusion plays an important role in the treatment of degenerative disorders of the spine and may also be appropriate for some cases of spine fractures, scoliosis, tumors, spondylolisthesis, facet joint pain, discogenic pain, and lumbar spondylosis. ${ }^{21}$ The chances of successful lumbar spinal fusion are increased significantly by the addition of a rigid fixation device. In a prospective randomized study of 124 patients undergoing lumbar or lumbosacral fusion, Zdeblick ${ }^{20}$ showed that rigid fixation in which instrumentation was used in addition to autogenous bone graft resulted in successful fusion in $95 \%$ of patients, compared with a rate of only $65 \%$ in patients without rigid fixation.

Unfortunately, there are significant complications associated with open instrumentation techniques. These are caused in part by the amount of exposure usually required to perform these procedures, and the significant muscle dissection (with its inherent denervation of the erector spinae muscles) needed for sufficient visualization of the osseous elements. Direct complications attributable to the approach used in these procedures are numerous. First, a mean of more than $500 \mathrm{ml}$ of intraoperative blood loss is common in lumbar fusion procedures, and this amount rises as the number of segments undergoing fusion increases. ${ }^{7}$ Transfusion of blood products places patients at risk for administrative errors (that is, ABO incompatibility), transfusion-related acute lung injury, and viral infections. ${ }^{3,4}$ Blood loss and the need for transfusions appear to be less common when minimally invasive techniques are used. ${ }^{5}$ Second, the degree and duration of muscle retraction is directly correlated with muscle injury. As may be expected, muscle damage has 

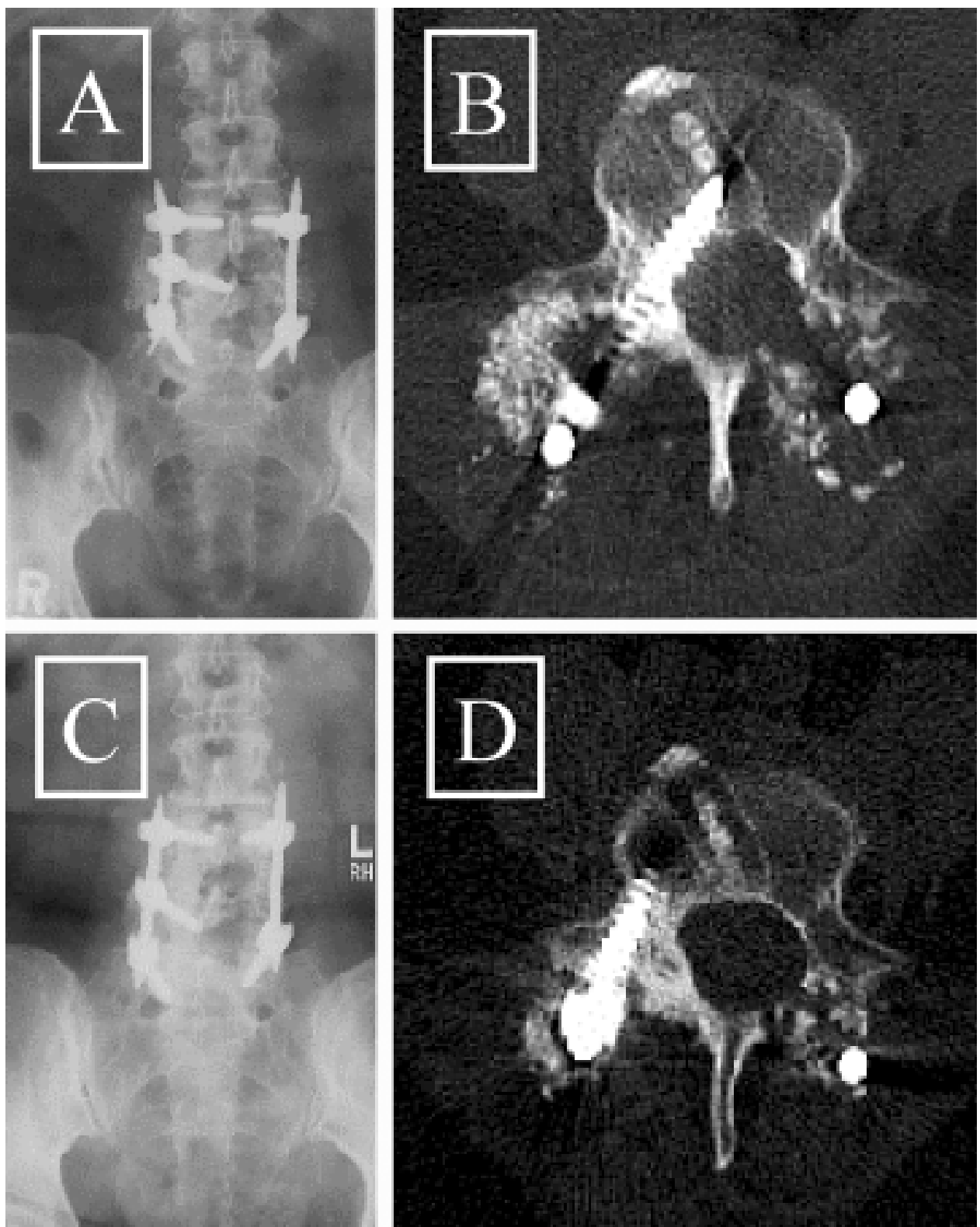

FIG. 3. Postoperative X-ray film, AP view (A) and axial CT scan (B) revealing an incidentally found medially breached PS. After slight repositioning surgery, the repeated AP x-ray film (C) and axial CT scan (D) reveal better placement.

been shown to be more common in larger spine procedures, because a greater degree and longer duration of muscle retraction is required for more extensive fusions. ${ }^{8,9}$ Pedicle screw instrumentation placed using an open technique remains the gold standard for posterior segmental fixation. As we become progressively more experienced with minimally invasive procedures, a relatively high accuracy of placement can be assured for various techniques (that is, the Gaines funnel technique, mammillary process, fluoroscopy, imaging guidance, and so on). Studies which have addressed the relative accuracy of placement, and specifically the screw malpositioning rates, have revealed a very high accuracy in series in which hundreds of screw placements have been reported. ${ }^{10,11,17}$ These have been confirmed radiographically, clinically, and sometimes by the results of CT scans. Although the literature contains reports by many authors detailing their understanding of screw malpositioning rates for fixation performed using open methods, the same cannot be said for percutaneously placed instrumentation. No paper has yet appeared in the literature that addresses this question specifically in a large number of percutaneously placed screws. In the present study we attempt to address the question of accuracy in the positioning of consecutive percutaneous PSs when they are placed with the aid of standard fluoroscopic guidance (without imaging guidance) in a reasonably large number of patients.

Although their work has not been published in the literature, several groups early on presented in abstract form series of small numbers of patients with PS placements in which this question was specifically addressed. Nockels 

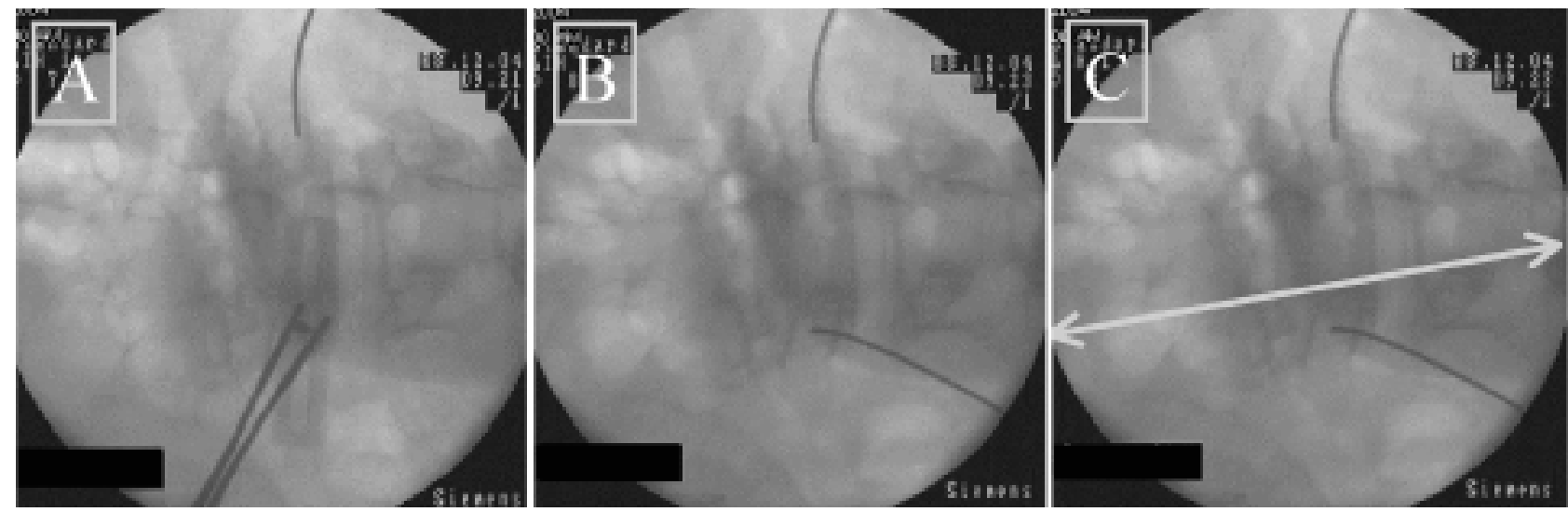

FIG. 4. Fluoroscopy study demonstrating the "owl's eye" technique. By placing the x-ray gantry in line with the pedicle and then orienting the Jamsheedi needle to this alignment (A), a K-wire can be safely placed through the middle of the pedicle (B). When the medial aspect of the pedicle of S-1 is not readily apparent, note that the location of the medial aspect of the pedicles of L-4 and L-5 can be used to determine it (C). The line with arrows indicates the middle aspects of the pedicles of L4-S1.

presented a report at the Joint Section on Disorders of the Spine and Peripheral Nerves in 2002 detailing placement of 64 screws, none of which were malpositioned. Perez-Cruet and colleagues presented a paper 1 year later in the same forum; they examined 100 cases of PS placement in the lumbar spine only. Of these 100 screws, four were malpositioned, yielding a $4 \%$ inaccuracy rate. The patients all underwent CT scanning to help assess the rate of screw malpositioning.

Historically, a method similar to current procedures for percutaneous PS instrumentation was used in large numbers of patients undergoing external fixation. Several studies have been published, the largest of which indicates a screw malpositioning rate of approximately $6.6 \%$, with problems most commonly occurring at $\mathrm{S}-1$, where the rate can exceed $12 \%$. Several published reports on permanently placed percutaneous PS instrumentation reveal malpositioning rates in smaller numbers of screws. We previously reported on 20 consecutive patients who underwent placement of percutaneous PS instrumentation, and we specifically addressed the short-term complications of this technique. ${ }^{5}$ No screw was malpositioned in this earlier series, and all were placed from L-3 to the sacrum.

Schwender, et al., ${ }^{16}$ placed percutaneous instrumentation in 49 patients undergoing transforaminal lumbar interbody fusion, two $(4.1 \%)$ of whom required screw repositioning, indicating a clinically significant malpositioning rate of approximately $1 \%$. Prior to this, in one of the first reported series of percutaneous hardware positioning in which a percutaneous rod system was used, a very high level of accuracy was found by a similar group of surgeons in the first 10 patients undergoing this technique. Other groups have also reported high rates of accuracy in minimally invasive PS placement when small numbers of screws are placed. . $^{6,12}$

In this study we identified only one instance of PS malpositioning in nearly 300 placements, for a $0.35 \%$ PS malpositioning rate. This one breach was found incidentally on a CT scan obtained for routine follow up (not based on symptoms), which highlights the disadvantage of using clinical criteria to determine the need for CT scanning for assessment of the breach rate. Seen in this light, one could criticize this paper because essentially only half of the screws were assessed with CT scanning to determine the breach rate. Conversely, one could question the clinical relevance of a minimally breached screw that is not causing symptoms. Regardless, in this series, when looking only at those screws that were placed in patients who later underwent CT scanning (which covered the entire spectrum of screws studied, T7-S1) or when looking at the entire series, a relatively low breach rate was found, comparing favorably with large published series of PS placement.

Although a relatively low PS breach rate has been shown, other published and presented data would perhaps call into question these highly accurate results. Khoo presented PS stimulation data to the Joint Section on Disorders of the Spine and Peripheral Nerves in 2003 in which $30.5 \%$ of the screws required repositioning after percutaneous placement of instrumentation (unpublished data). In $11.1 \%$ of cases, although fluoroscopy studies confirmed placement, both electromyography and postoperative CT scanning showed otherwise. Salerni ${ }^{15}$ described a minimally invasive technique for PS removal in his article. In this small series of patients, four underwent removal of the devices subsequent to symptomatic percutaneous PS malpositioning. Although the total rate of PS malpositioning cannot be calculated from this paper (the denominator, the total number of screws placed, is lacking), this represents a larger number of screw malpositions than has been reported in any series in the literature. These articles not only highlight the potential difficulty with percutaneous instrumentation, but also identify potential mechanisms to avert or treat it.

\section{Placement of Screws at the S-1 Level}

When performing percutaneous screw placement at S-1, the surgeon consistently finds that this level is the most difficult one in which to fix an instrument (L. T. Khoo, unpublished data) ${ }^{19}$ despite the size of the vertebra relative to those found in the lumbar spine. ${ }^{1}$ To limit the rate of medial breach at this level (0\% of 50 screws placed), several techniques have been adopted in our practice. These include the owl's eye technique of sighting in line with the pedicle 
with the aid of fluoroscopy rather than using the AP fluoroscopic angle; this technique is useful because one is constantly aware of the medial pedicular wall. In cases where the pedicle is not readily apparent, unless there is a significant deformity present, connecting the medial aspect of the pedicle with the levels above will help identify the medial aspect of S-1. By not allowing the initial entry point of the $\mathrm{K}$-wire even to approach this medial pedicle line, one ensures that the entry point is appropriately lateral. The pedicle at this level has the steepest medial angulation, which only serves to decrease the medial breach rate. Therefore, placement can be made based on other radiographic features when required (for example, the superior endplate of S-1 observed on the Ferguson view and the medial border of the pedicles at L- 4 and L-5 seen on the oblique view [Fig. 4]).

\section{Conclusions}

Percutaneous PS instrumentation can be safely placed throughout the thoracolumbar spine, with an overall low rate of pedicular breach $(0.35 \%)$ comparable with other large series of open PS placement. Furthermore, as the comfort level and experience with this procedure increases, one can safely place percutaneous instrumentation not only for standard degenerative disease, but also in patients with a significant sagittal, coronal, or rotational deformity. This technique can also be used for spinal reconstruction for tumor or trauma, or in cases of distorted anatomy resulting from previous surgical exposure.

\section{References}

1. Benzel EC: The essentials of spine biomechanics for the general neurosurgeon. Clin Neurosurg 50:86-177, 2003

2. Foley KT, Gupta SK: Percutaneous pedicle screw fixation of the lumbar spine: preliminary clinical results. J Neurosurg 97: $7-12,2002$

3. Goodnough LT: Risks of blood transfusion. Crit Care Med 31: S678-S686, 2003

4. Goodnough LT: Risks of blood transfusion. Anesthesiol Clin North America 23:241-252, 2005

5. Isaacs RE, Podichetty VK, Santiago P, et al: Minimally invasive microendoscopy-assisted transforaminal lumbar interbody fusion with instrumentation. J Neurosurg Spine 3:98-105, 2005

6. Jang JS, Lee SH: Minimally invasive transforaminal lumbar interbody fusion with ipsilateral pedicle screw and contralateral facet screw fixation. J Neurosurg Spine 3:218-223, 2005
C. J. Powers, V. Podichetty, and R. E. Isaacs

7. Johnson RG, Murphy M, Miller M: Fusions and transfusions. An analysis of blood loss and autologous replacement during lumbar fusions. Spine 14:358-362, 1989

8. Kawaguchi Y, Matsui H, Tsuji H: Back muscle injury after posterior lumbar spine surgery. A histologic and enzymatic analysis. Spine 21:941-944, 1996

9. Kawaguchi Y, Matsui H, Tsuji H: Back muscle injury after posterior lumbar spine surgery. Part 2: Histologic and histochemical analyses in humans. Spine 19:2598-2602, 1994

10. Kim YJ, Lenke LG, Bridwell KH, et al: Free hand pedicle screw placement in the thoracic spine: is it safe? Spine 29:333-342, 2004

11. Laine T, Lund T, Ylikoski M, et al: Accuracy of pedicle screw insertion with and without computer assistance: a randomised controlled clinical study in 100 consecutive patients. Eur Spine J 9:235-240, 2000

12. Maciejczak A, Barnas P, Dudziak P, et al: Minimalnie inwazyjna korpektomia tylna odcinka ledzwiowego kregoslupa. Neurol Neurochir Pol 38:511-517, 2004

13. Magerl FP: Stabilization of the lower thoracic and lumbar spine with external skeletal fixation. Clin Orthop Relat Res 189:125-141, 1984

14. Manek NJ, MacGregor AJ: Epidemiology of back disorders: prevalence, risk factors, and prognosis. Curr Opin Rheumatol 17:134-140, 2005

15. Salerni AA: Minimally invasive removal or revision of lumbar spinal fixation. Spine J 4:701-705, 2004

16. Schwender JD, Holly LT, Rouben DP, et al: Minimally invasive transforaminal lumbar interbody fusion (TLIF): technical feasibility and initial results. J Spinal Disord Tech 18:S1-S6, 2005

17. Smorgick Y, Millgram MA, Anekstein Y, et al: Accuracy and safety of thoracic pedicle screw placement in spinal deformities. J Spinal Disord Tech 18:522-526, 2005

18. Wiesner L, Kothe R, Ruther W: Anatomic evaluation of two different techniques for the percutaneous insertion of pedicle screws in the lumbar spine. Spine 24:1599-1603, 1999

19. Wiesner L, Kothe R, Schulitz KP, et al: Clinical evaluation and computed tomography scan analysis of screw tracts after percutaneous insertion of pedicle screws in the lumbar spine. Spine 25:615-621, 2000

20. Zdeblick TA: A prospective, randomized study of lumbar fusion. Preliminary results. Spine 18:983-991, 1993

21. Zdeblick TA: The treatment of degenerative lumbar disorders. A critical review of the literature. Spine 20:126S-137S, 1995

Manuscript received January 17, 2006.

Accepted in final form February 21, 2006.

Address reprint requests to: Robert E. Isaacs, M.D., Division of Neurosurgery, Duke University Medical Center, Box 3807, Durham, North Carolina 27710. email: robert.isaacs@ duke.edu. 